\title{
Training rats to discriminate between the odors of individual conspecifics
}

\author{
HEATHER MACINTOSH SCHELLINCK and RICHARD E. BROWN \\ Dalhousie University, Halifax, Nova Scotia, Canada \\ and \\ BURTON M. SLOTNICK \\ The American University, Washington, D.C.
}

\begin{abstract}
To determine the influence of genetic and microbiological factors in producing individually unique urine odors, we used a go/no-go operant task to assess the ability of eight male Long-Evans rats to discriminate between urine odors from the following pairs of male rats: (1) two outbred LongEvans rats, (2) individual conventionally housed rats differing genetically only at the major histocompatibility complex (PVG vs. PVG.R1), (3) individual PVG versus PVG.R1 rats born by cesarean section and raised in germfree conditions, and (4) conventionally housed, genetically identical individuals of the PVG or PVG.R1 strains. Discriminable differences were present between the urine odors used in all four tasks. Analysis of the errors to a criterion of $85 \%$ correct discrimination responses revealed different learning patterns in each task. When bacteria were not present in genetically different rats (Task 3 ) and when bacteria were present but there were no genetic differences between rats (Task 4), the discrimination was the most difficult. The easiest discrimination involved the presence of bacteria and a genetic difference at the major histocompatibility complex (Task 2). These results indicate that, although bacteria are not necessary for the production of discriminable odor differences, they do influence the discriminability of the urine odors of rats.
\end{abstract}

Urine odors provide individual animals with a unique identity (Brown, 1979; Halpin, 1986), but the origin of these odors of individuality has yet to be determined. Hormonal variations, dietary influences, and state of health contribute to an individual's odor, but these influences lack the stability required to create an odor that is constant over time within an individual yet differs consistently among individuals.

The sense of smell is highly developed in many species of animals. For example, Pfaff and Pfaffman (1969) have shown that male rats can detect one part of estrous urine in $10^{3}$ parts of water. Rats can also be trained to discriminate between concentrations of amyl acetate that differ by only $0.2 \%$ (Slotnick \& Ptak, 1977). Moreover, behavioral preference tests have shown that a number of mammals, ranging from sheep to mice, can discriminate between the urine odors of opposite-sex conspecifics that

We wish to thank Heather Duncan, University of Cincinnati Medical Center, for her valuable assistance in developing this project. We would also like to thank Terry Pendry, MRC Experimental Embryology and Teratology Unit, Surrey, England, and Jeff Herbert, Quadrant Research Foundation, Cambridge, England, for collecting the samples from the MHC urine donors. This research was supported by the Natural Sciences and Engineering Research Council of Canada Grant A7441 to R. Brown, and was undertaken by $\mathbf{H}$. Schellinck in partial fulfillment of the requirements for a Master of Science degree at Dalhousie University. H. Schellinck was supported by a postgraduate scholarship from the Natural Sciences and Engineering Research Council of Canada. Correspondence should be addressed to Richard E. Brown, Department of Psychology, Dalhousie University, Halifax, Nova Scotia B3H 4J1, Canada. differ only in levels of reproductive hormones (see Brown \& MacDonald, 1985, for a review). Such evidence indicates that animals can detect subtle variations in the odors of other individuals. Thus, to recognize an individual by its odor, they must be able to attend to an odor cue that is constant and learn to ignore more variable cues.

Recently, it has been suggested that specific genetic loci and commensal bacterial flora may provide a consistent mechanism for the production of individually distinct odors (Singh et al., 1990). A genetic basis for the individuality signals in the urine odors of mice and rats has been assigned to the genes of the major histocompatibility complex (MHC). These MHC genes code for glycoproteins that are incorporated into the cell membrane and provide the context for the recognition of foreign antigens by T lymphocytes (Klein, 1986). Since the MHC contains the most polymorphic loci known, it is unlikely that two nonidentical individuals will have the same MHC genes (Klein, 1986). Consequently, it is conceivable that the MHC glycoproteins could produce a unique individual odor.

The results of behavioral experiments with both mice and rats support this hypothesis. Mice have been trained in a $Y$ maze to discriminate between the odors of mice of the same sex that differ only at the MHC (Yamaguchi et al., 1981; Yamazaki, Beauchamp, Bard, Thomas, \& Boyse, 1982). In a habituation-dishabituation task, rats can discriminate between the urine odors of male rats that differ genetically only at the MHC Class I genes (Brown, Singh, \& Roser, 1987). Genetic differences only at the MHC also influence mate choice (Yamazaki et al., 1976; 
Yamazaki, Yamaguchi, Andrews, Peake, \& Boyse, 1978) and the incidence of pregnancy block in mice (Yamazaki et al., 1986; Yamazaki et al., 1983).

Brown et al. (1987) investigated the manner in which the variability in these cell surface proteins could be expressed in the urine. They determined that intact Class I MHC molecules purified from the urine of MHC congenic male PVG and PVG.R1 rats could not be discriminated. The remainder fraction of urine with the Class I molecules removed could, however, still be discriminated. Since gel electrophoresis (SDS-PAGE) indicated that the Class I molecule fragmented in the urine (Singh, Brown, \& Roser, 1987), Brown et al. (1987) suggested that a fragment of the MHC molecule, either by itself or in association with another compound, might be responsible for individually distinct urine odors. Furthermore, because rats could not discriminate between urines from PVG and PVG.R1 rats after the volatiles were removed by nitrogen purging, it was concluded that the MHC-related odor was produced by a volatile component of the urine (Singh, Brown, \& Roser, 1989).

Commensal bacteria that inhabit the gut of an animal are known to produce volatile metabolites, which are excreted in the urine (Scheline, 1973). To determine if the volatile component responsible for individual odor was bacterially derived, Singh et al. (1990) collected urine from PVG and PVG.R1 individuals born by cesarean section and raised in a germfree environment. Male rats tested in a habituation-dishabituation task could not discriminate between the urine odors from germfree animals of these two strains. When the germfree rats were moved to conventional housing after being inoculated with commensal flora, however, the urine from individuals of the two strains could be discriminated. These results suggest that commensal bacteria are essential for the production of the unique individual odor in the urine of MHC congenic rats.

The habituation-dishabituation procedure (adapted from Sundberg, Doving, Novikov, \& Ursin, 1982) consists of two phases: (1) the habituation phase, which involves repeated presentations of one odor until the subject habituates to that stimulus, and (2) the dishabituation phase, in which samples of a second odor are presented. The measurement of the change in behavior that occurs upon presentation of the second odor has been used as a criterion for determining olfactory discrimination. One problem with using the habituation-dishabituation task, however, is the difficulty of interpreting a result that indicates that there is no difference between two odors, such as that found by Singh et al. (1990).

The lack of investigation of an odor may indicate that the odor is indiscriminable from the previous sample, but it may also mean that the odor is not interesting to the animal, that the animal is not motivated to investigate the odor, or even that the odor is aversive and thus is avoided. The uncertainty created by such an ambiguous result may be resolved through the use of operant conditioning tech- niques. In this paradigm, the subject is motivated to respond, and its ability to learn may be quantified. Consequently, one can say for certain whether the subjects can or cannot discriminate between the odors. In addition, when the operant chamber is combined with a computercontrolled odor-delivery system (olfactometer), the stimulus can be precisely controlled and the animal's responses are automatically recorded.

The present experiment was designed to use an operant olfactometer to examine the ability of rats to discriminate between urine odors from outbred Long-Evans rats, conventionally housed MHC congenic individuals, germfree individuals of MHC congenic strains, and different individuals of the same inbred strain of rats. If the urine odors from individuals raised in germfree conditions are not discriminated, this would confirm the results reported by Singh et al. (1990) and overcome the ambiguity of the results found by using the habituationdishabituation task.

\section{METHOD}

\section{Subjects}

Nine male Long-Evans rats were purchased from Charles River Canada Inc., Quebec, at 90 days of age and housed individually in $48 \times 27 \times 16 \mathrm{~cm}$ polycarbonate cages with stainless steel grid tops and softwood chip bedding. The animals were kept on a reversed 12:12-h light:dark cycle (lights off at $8 \mathrm{a} . \mathrm{m}$.) and were provided with Purina Rat Chow and water ad lib. Two weeks prior to training, water availability was limited to $10-15 \mathrm{ml}$ per day. This waterrestriction schedule was continued for the duration of the experiment.

\section{Urine Donors}

Outbred rats. Urine was collected individually from 5 adult male outbred Long-Evans rats for $8 \mathrm{~h}$ per day during the dark phase of the light:dark cycle for 3 weeks. Each donor was housed in the same type of cage as the test subjects, except during urine-collection periods when they were housed in a $24 \times 19.5 \times 18.5 \mathrm{~cm}$ metabolism cage. Urine donors had free access to Purina Rat Chow and water, except during the 8-h urine-collection periods when only water was available.

Urine was collected in a gauze-covered glass bottle placed directly beneath the metabolism cage. The urine was removed from the collection bottles, labeled with the donor's number and the collection date, and stored in 2-ml aliquots at $-20^{\circ} \mathrm{C}$.

MHC congenic rats. Urine was collected from 5 individual male rats of each of the MHC congenic strains: PVG and PVG.RI. The PVG and the PVG.R1 strains differ only in the Class $1 \mathrm{~A}$ region of the MHC (Butcher \& Howard, 1986).

These animals were born in a specific pathogen free (SPF) unit at the Institute of Animal Physiology and Genetics Research, Babraham, Cambridge, England, and were moved to conventional housing at Quadrant Research Foundation, Cambridge, England, before urine collection. They were housed individually in $23.2 \times 35.2 \times 18 \mathrm{~cm}$ opaque plastic cages (NPK Ltd., U.K.) with free access to food (Special Diet Services irradiated RM3 cubes) and sterile water.

When these animals were 90-120 days of age, urine was collected for $8 \mathrm{~h}$ per day in a Urimax metabolism cage and then stored in a vial that was labeled with the number of the donor and the collection date. The urine was frozen at $-20^{\circ} \mathrm{C}$ until sufficient quantities were collected for shipment to our laboratory at Dalhousie University. It was then packed in dry ice in a Styrofoam container 
and flown to Halifax via Air Canada Cargo. It was again stored at $-20^{\circ} \mathrm{C}$ until it was used as an odor stimulus.

Germfree MHC congenic rats. Urine was collected from 5 PVG and 5 PVG.R1 male rats that were born by cesarean section to mothers from the SPF unit, Babraham, Cambridge, England, and reared in germfree isolators at the MRC unit, Carshalton, Surrey, England (Pendry, 1984). These rats were housed in sterile plastic boxes in groups of 4 , except during urine collection when they were placed in individual Urimax metabolism cages within the germfree units. Irradiated sterilized water and food (Special Diet Services irradiated RM3 cubes) were available ad lib (see Singh et al., 1990).

These rats were checked for bacterial sterility by nasopharyngeal swab at $\mathbf{1 4 0}$ days of age, just prior to urine collection, and found to be germfree. The urine collected from each rat was stored in plastic vials, labeled with the rat's identification number and the collection date, and stored at $-20^{\circ} \mathrm{C}$ until it was shipped to our laboratory at Dalhousie University.

\section{Apparatus}

Olfactometer. Odor stimuli were delivered by a computercontrolled olfactometer (Figure 1) modified from the basic design developed by Slotnick and Nigrosh (1974). Clean air was provided for the olfactometer by a continuous-duty GAST compressor pump [1] (numbers in square brackets refer to components of the olfactometer presented in Figure 1) and filtered through activated charcoal [2] and two fritted glass filters [3]. The rate of airflow into the olfactometer was regulated by Kontes $\# 2$ needle valves [4] and measured by Porter Instrument Co. flowmeters. A main air flow- meter [5] (Model B-125-50) monitored the clean airstream (4,000 cc/min); the other four flowmeters [6] (Model A-125-7) measured the flow of air to each of four stimulus channels $(120 \mathrm{cc} / \mathrm{min})$. The remainder of the olfactometer consisted of a system of glass and Teflon ductwork and connectors [7], [12], and [14], and General Valve Co. Teflon body series 1 and 2 solenoid valves [9], [13], and [16], which controlled the delivery of clean air and odorants to the odor-sampling tube [10] of the animal test chamber [11].

Clean air was delivered continuously to the test chamber through the common and normally open ports of the three-way solenoid final valve [9] (Model 1-30-900). The flow of air into each stimulus channel and release of odorant from each stimulus jar [15] was controlled by the action of two solenoid valves as follows: The common port of a three-way stimulus valve [13], upstream from the stimulus channel, was connected to the air flow from the stimulus channel flowmeter; the normally open port was connected to an exhaust line; the normally closed port was connected to the stimulus channel. When this three-way valve [13] operated, the clean air was diverted to the stimulus jar [15], where it became saturated with odorant. The flow of odorized air out of each stimulus jar and into the main airstream was controlled by the operation of a twoway solenoid valve [16] (Model 2-40-900). Coincident with the operation of the three-way valve [13], this two-way valve, downstream from the stimulus jar, opened and the odor-saturated air was introduced into a glass manifold [8] to join the main airstream.

The action of the two stimulus valves was synchronous with the closing of the final valve [9]. When the final valve closed, the clean airstream to the test chamber was briefly $(0.7 \mathrm{sec})$ sent to the ex-

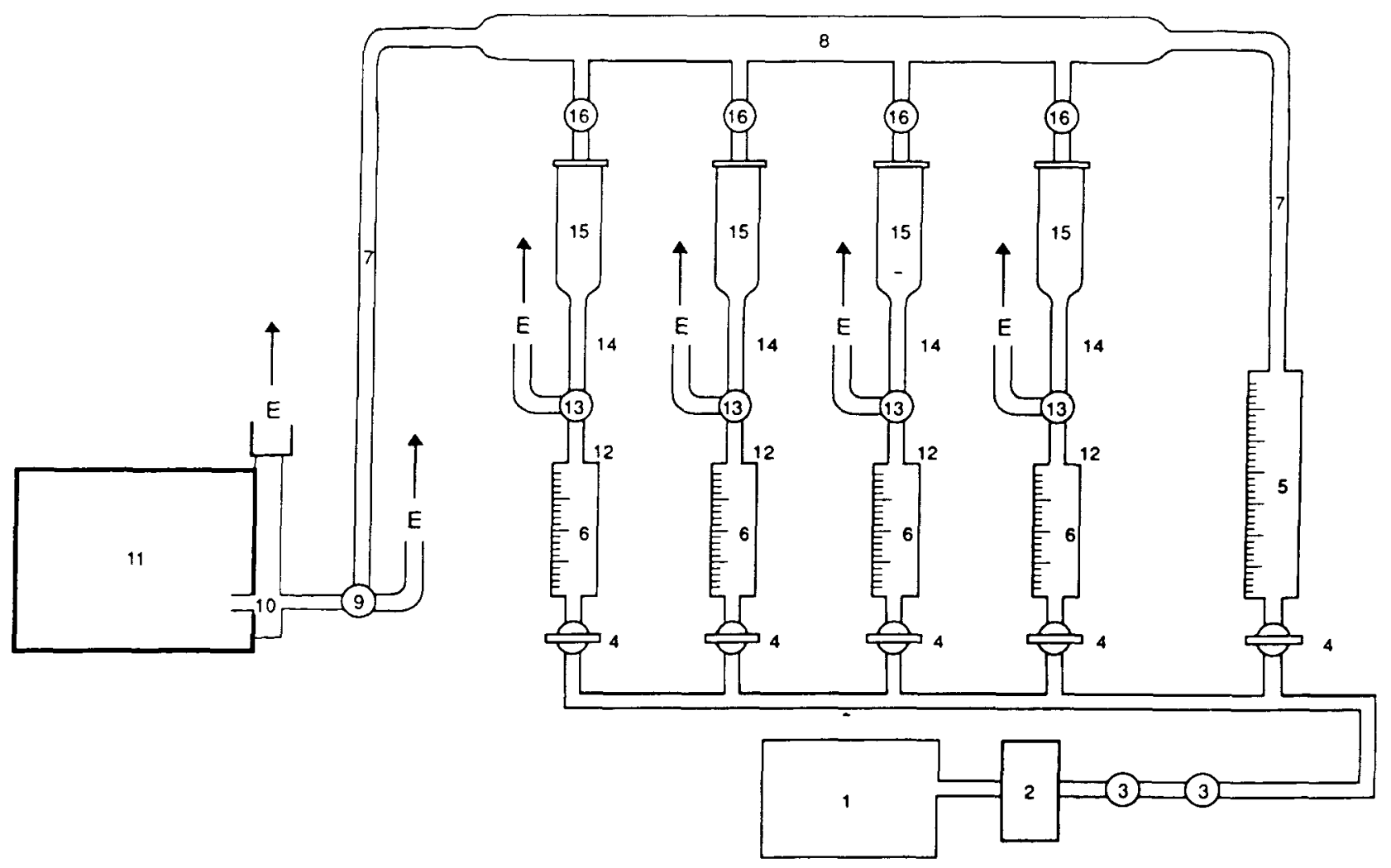

Figure 1. A schematic representation of the olfactometer. (1) GAST compressor pump, (2) activated charcoal filter, (3) fritted glass filters, (4) needle valves, (5) main air flowmeter, (6) stimulus channel fowmeters, (7) 8-mm glass ductwork, (8) glass manifold, (9) final valve, (10) odor-sampling tube, (11) animal test chamber, (12) Teflon connector, (13) three-way valve, (14) 4-mm glass ductwork, (15) odor stimulus jar, (16) two-way solenoid valve, $E=$ to exhaust. 
haust line, while the odor-saturated air mixed with the clean air in the manifold and was delivered to the final valve. When the final valve reopened, this odorized stream was presented to the test animal through the odor-sampling tube [10]. The airflow to the test chamber was exhausted to a ceiling vent through an exhaust line attached to the odor-sampling tube.

Animal test chamber. The animal test chamber [11] consisted of a $26.5 \times 17 \times 21.5 \mathrm{~cm}$ acrylic box with a stainless steel grid floor and a removable acrylic latch top. One side of the test chamber contained the odor-sampling tube [10] and a drinking tube (not shown in Figure 1).

The rat could sample the air delivered through the final valve by placing its nose through the opening in the odor-sampling tube. This nosepoke was detected by a photobeam located in the sampling tube. Water reinforcement was delivered to the rat via an 18-ga stainless steel drinking tube, which was wired to the grid of the floor to form a touch sensitive circuit. The rat's licking response completed this circuit and initiated delivery of $0.01 \mathrm{ml}$ of water from a reservoir controlled by a two-way solenoid valve.

\section{Procedure}

Preliminary training. Training rats to discriminate odors involved a preliminary training procedure in which the animals learned to obtain water reinforcement after breaking the photobeam and sampling the odor for a specific length of time. The training procedure was automated and controlled by an Apple Ile computer connected to the olfactometer by a digital interface (Bruce Field Inc.).

In the first training session, the subject was trained to obtain water by licking the water tube. In the next session, the rat was trained to poke its nose into the odor-sampling tube prior to receiving water reinforcement from the drinking tube. When a nosepoke broke the photobeam, a stimulus channel solenoid valve opened and a stimulus odor was presented. Urine collected from a male Long-Evans outbred rat was used as the training stimulus odor. The amount of time the subject was required to keep its nose in the sampling port was gradually increased. No reinforcement was available if the rat failed to break the photobeam for the required length of time.

In the next phase of this session, after the rat initiated a trial by breaking the photobeam, it was required to wait $0.25 \mathrm{sec}$ for the stimulus to be presented. During this period, the final valve and the stimulus valve operated simultaneously so that the main airstream containing the odorant was diverted to exhaust. This ensured that the odor mixed completely with the main airstream prior to its entry to the test chamber. The length of time that the final valve operated was gradually increased so that, in the last 20 trials of the session, the animal was required to wait $0.7 \mathrm{sec}$ before the odor was introduced for sampling. If the animal consistently withdrew its nose from the odor tube before the final valve had reopened, the previous time constraints were reintroduced until the animal learned to wait for the final valve to reopen and the odor to be delivered. Odor discrimination training began as soon as this session was completed.

Odor-no-odor discrimination training. The parameters in place at the end of the preliminary training procedure were also used during this stage. The rat received water reinforcement only after it broke the photobeam with a nosepoke, held its nose in the odorsampling port for $0.7 \mathrm{sec}$ while the main valve diverted air from the sampling tube, sampled the odor for a minimum of $0.25 \mathrm{sec}$, and then licked the drinking tube. The odor valve remained open for a maximum of $2 \mathrm{sec}$. No reinforcement was available during a 2 -sec interval immediately following the offset of the main valve and the presentation of the stimulus odor. In addition, a 4-sec intertrial interval was introduced. Figure 2 outlines the sequence of events for a positive stimulus odor $(S+)$ trial.

Urine collected from the same outbred Long-Evans male rat used in preliminary training provided the $S+$ for the discrimination training. The subjects were first presented with two blocks of $20 \mathrm{~S}+$ trials; then, no-odor (clean air) trials were introduced as the nega- tive stimulus trials $(\mathrm{S}-)$. The session continued with $100 \mathrm{~S}+$ and $100 \mathrm{~S}-$ trials presented in random order, with the following three constraints: (1) the session began with an $S+$ trial, (2) an equal number of $S+$ and $S-$ trials occurred in each block of 20 trials, and (3) in any one block, no more than three $S+$ or $S-$ trials were presented in succession (Slotnick \& Schoonover, 1984).

The $S+$ trials that terminated in reinforcement and the $S-$ trials in which the subject did not respond were scored as correct. The $\mathrm{S}+$ trials in which the animal sampled the stimulus but did not lick the drinking tube and $S$ - trials in which the animal did lick were scored as incorrect. A response on an $S$ - trial terminated the trial and initiated the intertrial interval. Two sessions of 240 trials were run with 40 introductory $S+$ trials given prior to the $200 \mathrm{~S}+$ and $S$ - trials.

The remainder of the sessions required to learn this odor-no-odor discrimination omitted the $S+$ only trials and began with $100 \mathrm{~S}+$ and $100 \mathrm{~S}-$ trials presented under the constraints listed above. When an animal scored a minimum of $85 \%$ on the first block of 20 trials and a mean of $85 \%$ or higher on the remaining nine blocks of the 200-trial session, it was considered to have met the criterion for successfully acquiring the discrimination. The data for this stage and all successive stages of the experiment were recorded through input to the Apple IIe computer from the digital interface.

Two-odor discrimination training. After successful completion of the odor-no-odor discrimination, the subjects were required to learn four different odor discrimination tasks. In Task 1 , the $S+$ and $S$ - odors were taken from two different donors from the outbred strain. The urine from one donor was the $S+$ for 4 of the subjects; the urine from the other donor was the $S+$ for the other 4 subjects. Different samples from the $S+$ odor donor and from the $\mathrm{S}-$ odor donor were used for each session. The criterion for learning was $85 \%$ correct as described above. In this task, and all successive tasks, the urine from each individual donor was always presented in the same stimulus jar; however, the stimulus jars were rotated systematically from one stimulus channel to another before each session.

In Task 2, the animal was required to discriminate the urine odors of conventionally housed male PVG rats from those of male PVG.R1 rats; these two strains differ only in the classical Class $1 \mathrm{~A}$ region of the MHC. The urine from one strain was designated the $S+$ for 4 of the experimental animals, and the urine from the other strain was the $S+$ for the other 4 , over all sessions. Urine from four individual donors of each strain was used for odor stimuli.

To be able to consistently discriminate between individuals, animals must be able to ignore the subtle day-to-day variations that might occur in the urine due to physiological and environmental changes. To determine if the subject's performance was influenced by a change in urine samples, more than one sample of $S+$ and $S-$ urine served as stimuli. All $S+$ samples were selected from animals of one strain, and all $S$ - samples were from animals of the other strain. If a change of urine samples within the $S+$ and the $S$ - set of donors does not produce a decrease in correct responses, then we can conclude that learning has generalized between samples of the same odor set. This would indicate that the subjects were not influenced by the day-to-day variations in individual odors in either the $S+$ or the $S-$ urine sample.

The method of introducing the second pair of $S+$ and $S$ - odors was as follows: One $S+$ sample and one $S$ - sample were presented for discrimination during the first 100 trials (Blocks 1-5) of each session, and the second pair of $S+$ and $S$ - samples was used for the remaining 100 trials (Blocks 6-10) of each 200-trial session. If the level of performance dropped on Block 6 after the betweenstrain discrimination had already been established, this would indicate that the subjects were now detecting differences that were not genetically based in the within-strain samples.

Three types of odor changes for both the $S+$ and for the $S-$ were tested. As outlined in Table 1, these changes were (1) two aliquots of the same urine sample collected from one donor animal (same 
1.

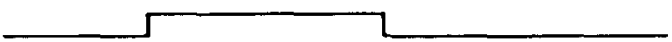

2.

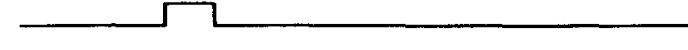

3.

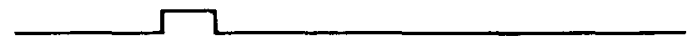

4.

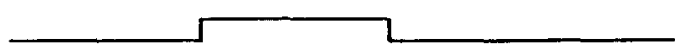

5.

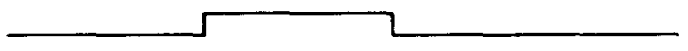

6.

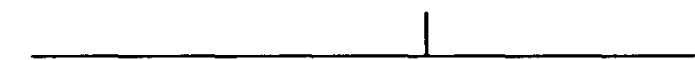

7.

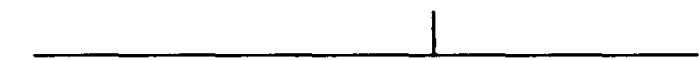

Water reinforcement

8.

$$
(\longmapsto=1 \mathrm{sec})
$$

Figure 2. The sequence of events during an $S+$ trial: (1) The subject initiates and maintains a photobeam break for a minimum of $0.95 \mathrm{sec}$; (2) the final valve diverts the airflow to exhaust for $0.7 \mathrm{sec}$; (3) the stimulus valve opens and an odor is diverted to exhaust for $0.7 \mathrm{sec}$; (4) the final valve operates and the odor is diverted to the sampling port for a maximum of $2 \mathrm{sec}$, during which (5) a 2 -sec fixed interval is in place when no reinforcement is available; (6) a licking response follows, resulting in (7) a $.02-\mathrm{ml}$ water reinforcement for a correct response; (8) a 4-sec intertrial interval follows the end of a trial.

Table 1

The Three Types of Within-Strain Odor Change for the $\mathbf{S}+$ and for the $\mathbf{S}-$ Between Blocks 5 and 6 on Tasks 2 and 3

\begin{tabular}{|c|c|c|}
\hline & Blocks 1-5 & Blocks 6-10 \\
\hline Same donor, same sample & $\begin{array}{l}\text { PVG }_{1} \text {, Sample } 1(\mathrm{~S}+) \\
\text { vs. } \\
\text { PVG.R1, Sample } 1(\mathrm{~S}-)\end{array}$ & $\begin{array}{l}\text { PVG }_{1} \text {, Sample } 1(\mathrm{~S}+) \\
\text { Vs. } \\
\text { PVG.R } 1_{1} \text {, Sample } 1(\mathrm{~S}-)\end{array}$ \\
\hline Same donor, different samples & $\begin{array}{l}\text { PVG }_{2} \text {, Sample } 1(S+) \\
\text { vs. } \\
\text { PVG.R1 } 1_{2} \text {, Sample } 1\left(S_{-}\right)\end{array}$ & $\begin{array}{l}\text { PVG }_{2} \text {, Sample } 2(S+) \\
\text { Vs. } \\
\text { PVG.R1 } \\
\text {, Sample } 2\left(S^{-}\right)\end{array}$ \\
\hline Different donors & $\begin{array}{l}\mathrm{PVG}_{3}(\mathrm{~S}+) \\
\text { vs. } \\
\text { PVG.R1 } \\
\text { PV-) }\end{array}$ & $\begin{array}{l}\text { PVG }_{4}(\mathrm{~S}+) \\
\text { VS. } \\
\text { PVG.R14 (S-) }\end{array}$ \\
\hline
\end{tabular}

Note-In Task 2, the urines were from conventionally housed MHC congenic strains; in Task 3, the urines were from germfree MHC congenic strains. 


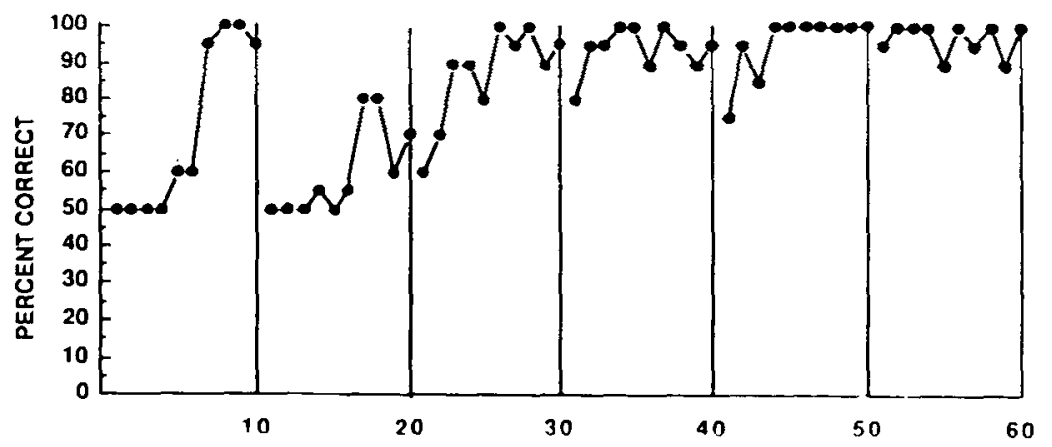

Figure 3. The learning curve for 1 subject in the discrimination of the urine odors from two outbred Long-Evans rats. A different sample was used for the $S+$ and $S-$ odors for each session.

donor, same sample), (2) two urine samples from the same donor animal collected on different days (same donor, different samples), and (3) two urine samples from different donor animals of the same strain (different donors).

Within the maximum of 10 sessions, the within-strain odor changes tested at midsession for each subject could include the following: (1) two sessions of same-donor samples for both the $S+$ and the $S-,(2)$ four sessions of same-donor/different-day samples for both the $S+$ and the $S-$, and (3) four sessions of differentdonor samples for both the $\mathbf{S}+$ and the $S-$. Criteria for learning the discrimination were $85 \%$ correct on the first block, a mean of $85 \%$ or more on the remaining nine blocks, and no decrease in percent correct below $85 \%$ between Blocks 5 and 6 .

Task 3 involved the discrimination of urine samples from PVG and PVG.R1 animals reared in germfree conditions. Criteria for learning the discrimination were identical to those described for Task 2. A similar midsession odor switch was also included in this task (see Table 1).

Task 4 involved the presentation of urine samples from animals of the same strain for discrimination. Three different types of odor stimuli were tested as follows: two aliquots of the same sample from an individual donor for a maximum of two sessions, two samples taken from the same individual on different days for a possible four sessions, and samples from two different individual donors for a maximum of four sessions. Four of the subjects were tested for their ability to discriminate between urine samples from PVG donors, and 4 were tested with urine from PVG.RI donors. Criteria for learning the discrimination were $85 \%$ correct on the first block and a mean of $85 \%$ correct on the remaining nine blocks.

After the subject reached the discrimination criterion or completed 10 sessions, it was immediately moved to the next task. Four rats were tested on the tasks in the following order: Task 1, Task 2, Task 3, and Task 4 . The other 4 were tested in the order: Task 1 , Task 3, Task 2, and Task 4.

\section{RESULTS}

\section{Sessions to Criterion}

On the basis of the criteria of $85 \%$ correct on the first block of 20 trials and a mean of $85 \%$ correct on the next four blocks, all 8 rats learned to discriminate between the urine odors of the two male Long-Evans rats (Task 1) and between the urine odors of conventionally housed males from the PVG and PVG.R1 strains (Task 2). Only 2 of the 8 subjects reached criterion in the discrimination between the urines of the germfree PVG and PVG.R1 rats
(Task 3). Both of these rats were inadvertently run on the same $\mathbf{S}$ - samples for two sessions in succession and, when tested with different urine samples in the following session, they did not reach criterion. None of the 8 rats learned the within-strain discrimination (Task 4) to criterion. Figures 3, 4, 5, and 6 show the learning curves for 1 subject in each of the four tasks. All other subjects showed similar learning curves.

The number of sessions that each animal required to meet the learning criterion are shown in Table 2. If the rat did not learn the task, it was given a score of 10 as the number of sessions to meet criterion. There was a significant overall difference in sessions to criterion over the four tests $[F(3,21)=42.27, p<.001]$. Post hoc (NewmanKeuls) tests showed that significantly fewer sessions were required to meet the criterion on Tasks 1 and 2 than on Tasks 3 and $4(p<.05)$. Sessions to criterion did not differ significantly between Tasks 1 and 2 or between Tasks 3 and 4.

\section{Errors to Criterion on Blocks 1-5}

An inability to meet the learning criterion in Tasks 3 and 4 did not mean that the subjects never performed

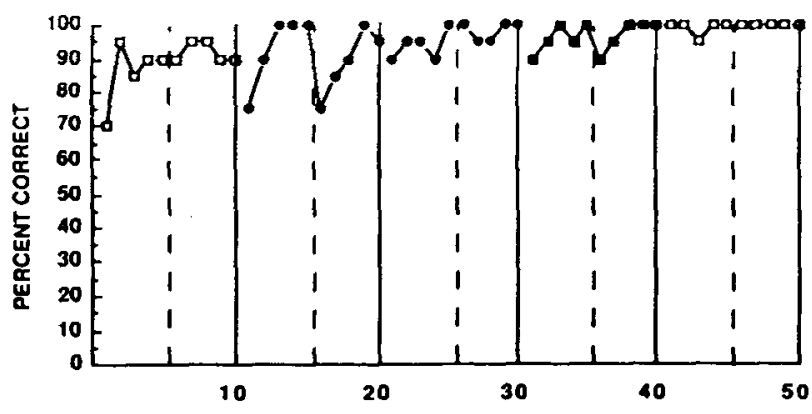

Figure 4. The learning curve for 1 subject in the discrimination of the urine odors from individual MHC congenic (PVG vs. PVG.R1) rats that were conventionally housed. After five blocks, both the $S+$ and the $S$ - odors were switched to different aliquots of the sample ( $\square$ ), to different samples from the same individual (๑), or to samples from different individuals $(\square)$. 


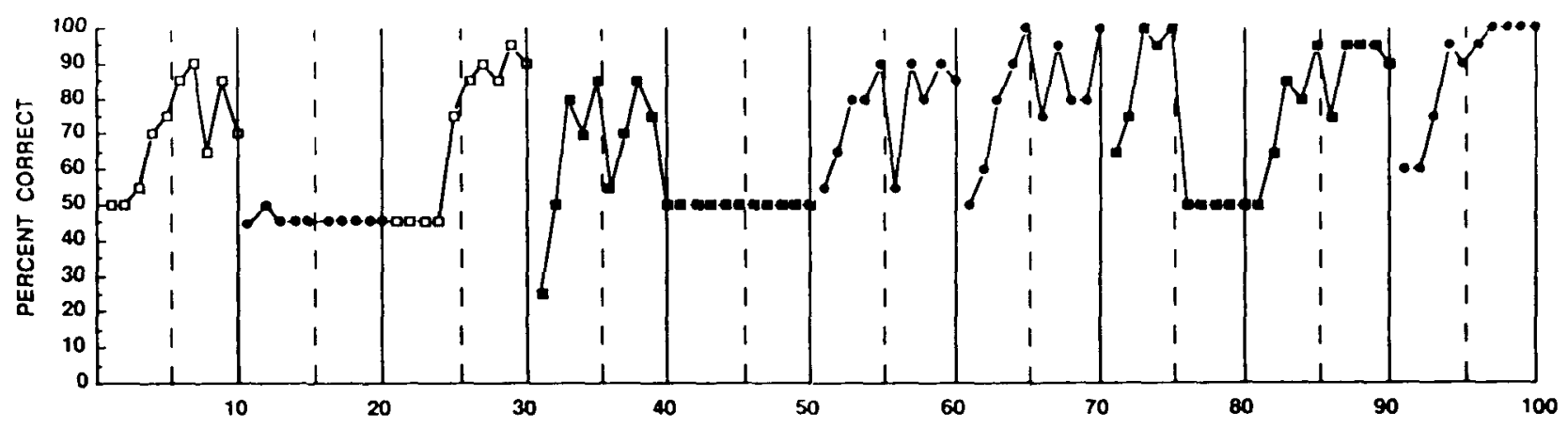

Figure 5. The learning curve for 1 subject in the discrimination of the urine odors from individual MHC congenic (PVG vs. PVG.R1) rats that were housed in germfree conditions. After five blocks, both the $S+$ and the $S-$ odors were switched to different alliquots of the same sample $(\square)$, to different samples from the same individual $(\bullet)$, or to samples from different individuals $(\square)$.

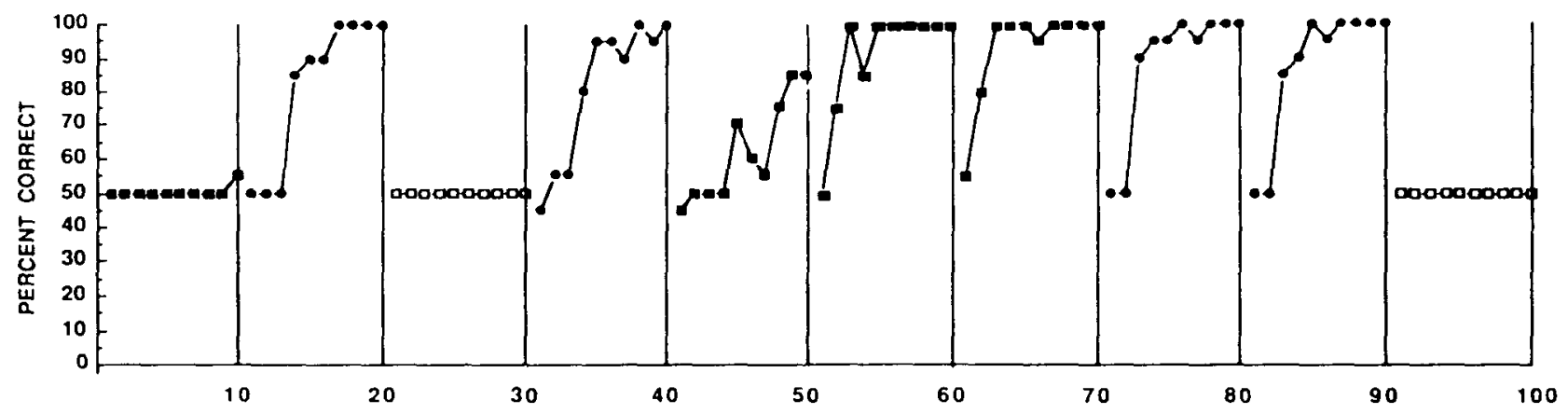

Figure 6. The learning curve for 1 subject in the discrimination of different aliquots of the same urine sample ( $\square$ ), difterent samples from the same individual (๑), or samples from two individuais of the same inbred strain (a) (e.g., PVG vs. PVG).

greater than chance in these tasks (see Figures 3-6). An analysis of the mean number of errors to criterion, or for all 10 sessions for the subjects not reaching criterion, indicated that the tasks varied in degree of difficulty (Figure 7). There was a significant overall difference in the mean number of errors over the four tasks $[F(3,21)=$ 73.17, $p<.001$ ]. Post hoc (Newman-Keuls) tests showed that the number of errors to criterion on Task 2 was significantly lower than the number of errors to criterion on Task $3(p<.01)$, Task $4(p<.01)$, and Task 1 $(p<.05)$. The number of errors to criterion on Task 1 were also significantly lower than the number of errors to criterion on Tasks 3 and $4(p<.01)$. The number of errors to criterion did not differ between Tasks 3 and 4 .

\section{Effect of Test Order}

Because the order in which the subjects received the germfree and conventional PVG and PVG.RI urines may have influenced the rate of learning, we compared the errors to criterion for the 4 animals tested on Task 2 prior to Task 3 and the $\mathbf{4}$ animals tested on Task 2 after Task 3. This indicated that the animals tested on Task 2 after Task 3 made significantly more errors on Task $2[t(6)=$ $2.9, p<.05]$, but there was no effect of test order on the number of errors to criterion in Task 3 . Although the number of errors on Task 2 differed depending on test order, a $t$ test for related samples indicated that the mean number of errors to criterion on Task 2 was significantly lower than the mean number of errors on Task 3 when Task 2 was presented before Task $3[t(3)=6.9, p<.01]$ and when Task 2 was presented after Task $3[t(3)=11.37$, $p=.001]$.

\section{Errors on Blocks 5 and 6}

As noted in Table 1, Tasks 2 and 3 required an additional learning criterion on Block 6 , when the $S+$ and $S-$ urine samples were switched. Analysis of the mean number of errors on Blocks 5 and 6 (Figure 8) revealed that, in the discrimination of urine from conventionally housed PVG and PVG.R1 rats (Task 2), there were no significant differences in the number of errors between Blocks 5 and 6 in any of the three types of odor change. All animals continued to meet the criterion of $85 \%$ correct on Block 6 , when the $S+$ and $S$ - odors were switched to different aliquots of the same samples used on the first five blocks. Similarly, performance did not drop when samples were taken from the same donor on different days. Seven of the 8 animals met criterion on Block 6 , 
Table 2

The Number of Sessions Required to Reach a Criterion of $85 \%$ Correct on Block 1 and $85 \%$ Correct on Blocks 2-4 for Each Subject for Each Discrimination

\begin{tabular}{|c|c|c|c|c|c|c|c|c|c|c|c|}
\hline & \multicolumn{8}{|c|}{ Subjects } & \multirow[b]{2}{*}{ Medium } & \multirow[b]{2}{*}{ Mean } & \multirow[b]{2}{*}{$S E M$} \\
\hline & Sheldon & Pembroke & Trinity & Dunston & Chelsea & Hampton & Magnus & Winchester & & & \\
\hline Task 1 & & & & & & & & & & & \\
\hline $\begin{array}{l}\text { Outbred Long-Evans } \\
\text { Task } 2\end{array}$ & 6 & 6 & 3 & 5 & 7 & 7 & 5 & 5 & 5.5 & 5.5 & .37 \\
\hline $\begin{array}{l}\text { Conventionally housed } \\
\text { PVG and PVG.RI }\end{array}$ & 2 & 2 & 2 & 3 & 6 & 7 & 2 & 7 & 2.5 & 3.86 & .94 \\
\hline $\begin{array}{l}\text { Task } 3 \\
\text { Germfree } \\
\text { PVG and PVG.R1 } \\
\text { Task } 4\end{array}$ & 10 & 10 & 6 & 10 & 10 & 10 & 9 & 10 & 10 & 9.38 & .63 \\
\hline $\begin{array}{l}\text { Within-strain } \\
\text { PVG or PVG.R1 }\end{array}$ & 10 & 10 & 10 & 10 & 10 & 10 & 10 & 10 & 10 & 10.00 & .00 \\
\hline
\end{tabular}

when the $S+$ and $S$ - samples were changed to different donors of the same strain.

In contrast, when the odors were from germfree PVG and PVG.R1 rats (Task 3), the animals' performance over the 10 sessions was inconsistent. There were no significant differences between the number of errors in Blocks 5 and 6 in Task 3, when the odors were changed to different aliquots of the same sample or to different samples from the same donor. Six of the 8 animals continued to score above criterion on different aliquots of the same sample after the odor switch; the other 2 animals had not reached criterion before the switch and did not improve on Block 6 after the switch. Five animals scored above criterion on different samples from the same donor. There were significantly more errors in Block 6 than in Block 5, when the $\mathrm{S}+$ and $\mathrm{S}-$ samples were changed to $\mathrm{S}+$ and $S-$ samples from different donors $[t(7)=2.94, p=.02]$; that is, the animals' performance was distupted by changing the $S+$ and $S-$ samples to other $S+$ and $S-$ samples from different individuals of the same strain in Task 3. One animal scored above criterion on samples from different donors; this occurred on one session. When tested with samples from different donors in three other sessions, this rat did not meet the criterion of $85 \%$ correct on both Blocks 5 and 6 (i.e., his performance was disrupted by the switch in odors).

\section{DISCUSSION}

These results allow us to conclude that both genetic differences at the MHC and commensal bacteria contribute to the unique odor of individuality found in the urine of rats. Although discriminable differences were present between the urine odors of individual Long-Evans male rats, conventionally housed PVG and PVG.R1 male rats, and bacteria-free PVG and PVG.R1 male rats, analyses of the errors to criterion revealed that the degree of difficulty of the tasks differed significantly depending upon the type of urines being discriminated. These differences in performance indicate differences in the type of cues available in the urines.
The ability of all subjects to meet the criterion for discrimination of the urines in Tasks 1 and 2 would suggest that, in these instances, a constant cue was used to discriminate the samples. The subjects made few errors and were able to retain the information from session to session. Because these tasks required a discrimination between the same two individuals (Task 1) or between a number of genetically identical individuals of one strain and a number of genetically identical individuals of another strain (Task 2), this result supports the hypothesis that genetic cues are involved in determining the individual odors of rats.

Further evidence that the discrimination of MHC congenic rats was based upon a constant odor cue was found

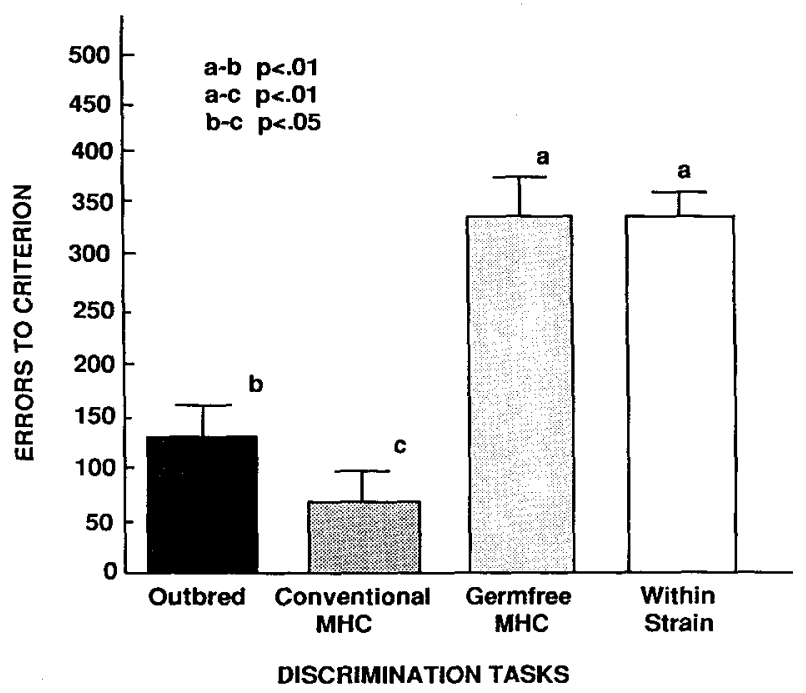

Figure 7. The mean number of errors $( \pm S E M)$ to a criterion of $85 \%$ correct on each of four odor discrimination tasks. Task 1: urine odors from two Long-Evans rats. Task 2: urine odors from conventionally housed MHC congenic rats. Task 3: urine odors from germfree MHC congenic rats. Task 4: urine odors from individuals within a single MHC inbred strain. Differences between groups from the results of a one-way ANOVA and Newman-Keuls post hoc tests. 


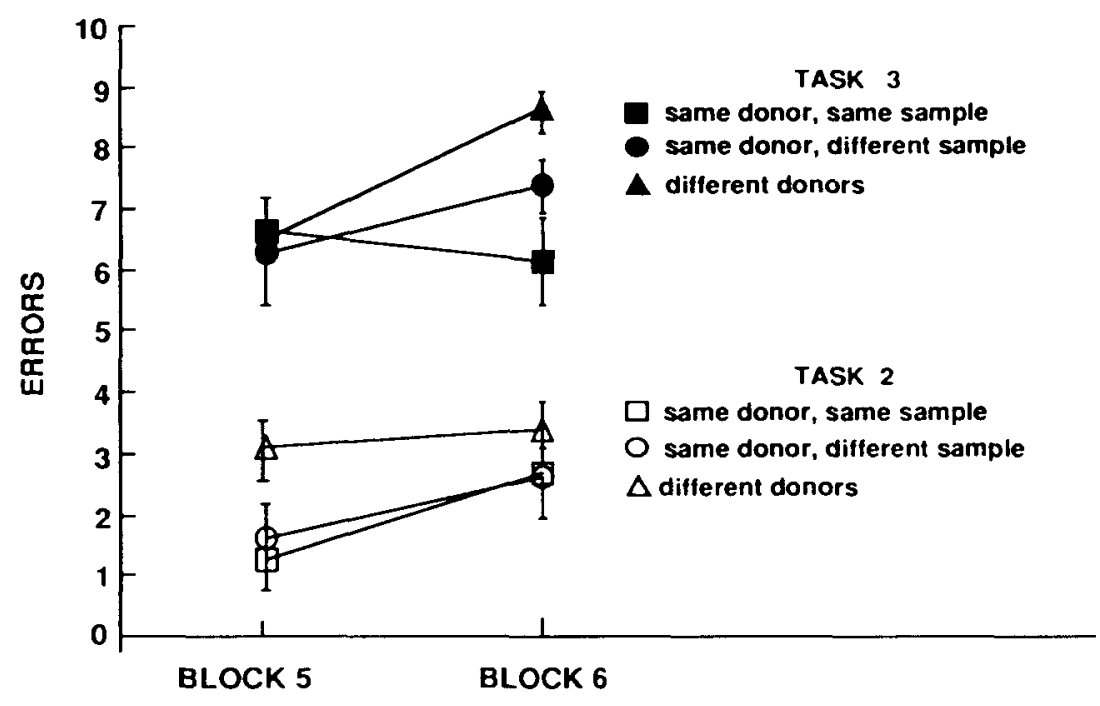

Figure 8. The mean number of errors $( \pm S E M)$ on Blocks 5 and 6 of Tasks 2 and 3. On Block 6, three different changes were made in the $S+$ and $S$ - odors: a different aliquot of the same sample presented on Block 5 , a different sample from the same donor used on Block 5, and a sample from a different donor of the same strain used on Block 5.

in the behavior of the animals after the $\mathrm{S}+$ and $\mathrm{S}$ - odors were changed to different samples of the same $S+$ and $S-$ strains as were used in the first five blocks of each session in Task 2. Some of the animals showed a decrement in performance at the beginning of Block 6 on the first session when the odors were switched; however, on the next session, they did not drop below the $85 \%$ correct criterion. This initial drop would indicate that they were aware of some differences in the within-strain samples but were subsequently able to ignore them. Seven of the 8 animals were able to meet criterion after all three types of odor switch (Table 1). In no instance did performance decline between Blocks 5 and 6, when the midsession change was made to different aliquots of the same sample. This would suggest that the discrimination continued to be based upon between-strain differences alone, and was not influenced by changing the samples within strains. Overall, these results indicate that the animals' performance was not influenced by differences, such as those created by hormones or their metabolites, other physiologically produced changes, concentration variations in the urine samples, or occasional contaminants in the urine.

The results in Tasks 1 and 2 contrast with those in Tasks 3 and 4 . In the latter tasks, even though there were some discriminable differences between the urine samples, the subjects made many errors and could not reach the criterion for learning the discrimination. When the discrimination was between urine odors from MHC congenic individuals raised in germfree conditions (Task 3), the subjects could learn to discriminate between one pair of samples in one session; however, in the next session, their performance indicated that they did not benefit from the information learned in the previous session (see Figure 5). Thus, it appears that each pair of samples was treated as a new discrimination. In other words, constant cues upon which to base a discrimination were not being found.

Further evidence that constant cues were not being used in Task 3 is found in the analysis of the errors made when both the $S+$ and the $S$ - odors were changed to other $S+$ and $\mathbf{S}$ - samples after five blocks as described in Table 1 . Although there was no decrement in performance at midsession, when the switch was to aliquots of the same sample or to samples from the same animal, there was a significant decrease in performance when the odor stimuli were changed to odors from different animals of the same strain. Thus, it would seem that a within-strain odor change interrupted the between-strain odor discrimination. Not only were different odor cues being used to discriminate between animals of different strains in Task 3, relative to those used in Task 2, but also a constant cue was not found between different animals of the same strain, again unlike the performance of the animals in Task 2.

In Task 4, when the discrimination was between genetically identical individuals or between samples taken from the same individual on different days, the subjects also relearned the task from session to session. However, as can be seen in Figure 6, the different aliquots of the same sample were not discriminable. Prior to running the experiment, it was not known if any of the within-strain samples would be discriminated beyond chance in Task 4, because there could not be any cues other than variable ones to base the discrimination upon. However, it would appear that the extreme sensitivity of rats to odor differences 
does enable them to discriminate between samples varying in such nongenetic components as hormone levels or their by-products, other metabolites, or possibly urine concentration. The exact nature of the cues used cannot be determined from this experiment.

In the only other reported examination of the ability of rats to discriminate between urine odors from genetically identical individual rats, Brown et al. (1987) found that samples from different individuals of the PVG.R1 strain, but not the PVG strain, could be discriminated in a habituation-dishabituation task. In a repetition of the same experiment, using other urine samples from new PVG.R1 and PVG donors, there was no longer a statistically significant difference in sampling time for the odors of different animals of the same strain. Brown et al. (1987) concluded that contaminants in the urine were probably responsible for the earlier discriminability of the samestrain individuals. Without chemical analysis of the particular samples involved, this suggestion cannot be confirmed. Concentration or chemical differences between the urine samples could be equally responsible.

Although it was possible for the animals to discriminate between the odors of genetically identical individuals, it should be noted that whatever difference was found between the samples in Task 4 could be ignored when it was no longer relevant to the task. For example, the discrimination in Task 4 was the reverse of the within-strain odor change in Task 2 when the subjects were required to ignore any noticeable differences between the two $S+$ samples and between the two $S-$ samples when the samples were changed in midsession. As noted previously, the rats' ability to disregard these changes in Task 2 but not in Task 3 indicates that constant cues were used for discrimination between the two congenic strains in Task 2 but not in Task 3.

A close examination of the possible differences between the two groups of urine donors might reveal why the test rats found Task 3 more difficult than Task 2 . Since PVG and PVG.R1 strains were the urine donors of the $S+$ and $S-$ odors in both Tasks 2 and 3, genetic factors cannot have contributed to the difference in performance on the two tasks. Due to the exacting requirements of maintaining the germfree status of the animals in Task 3, the food and water of these animals were irradiated and their environment kept sterile; however, other than maintaining the germfree status, it does not seem likely that these factors contributed to the difference between the urine odors on the two tasks.

In both tasks, it might be expected that nongenetic factors could contribute to metabolic, hormonal, or concentration differences and that these cues might be used if no other cues were present. However, there is no reason to believe that these changes would be consistent within the donors of one task or the other. Consequently, the only consistent factor that would probably contribute to the difference in performance between the two tasks would be the germfree status of the animals in Task 3. Thus, it would appear that bacteria are involved in the production of consistently discriminable odors, as hypothesized by Singh et al. (1990). They based their hypothesis on the results of a habituation-dishabituation task in which subjects did not show any discrimination between the odors of germfree PVG and PVG.R1 urines but did discriminate between the urines of conventionally housed MHC congenics.

In the present study, the subjects gradually learned to discriminate between the germfree urines and between samples from individuals of the same strain (Figures 5 and 6). Thus, the results are not entirely in agreement with the findings of Singh et al. (1990), although urine from the same germfree and conventionally housed donors was used in both studies. However, as previously discussed, the analysis of the number of errors on the first five blocks (Figure 4) confirmed a difference in the ability of the subjects to discriminate between the urine odors of conventionally housed MHC and germfree congenic individuals.

We would hypothesize that the type of task used in this experiment contributed to the different findings. The ability to discriminate between germfree individuals of different strains and between individuals of the same strain, in this instance, may be explained by the interaction of the rat's olfactory capabilities and the motivational state created by the water-restriction schedule required for the operant task. It would appear that, when highly motivated, the rat has the ability to discriminate between samples on the basis of the cues provided by the small variations always present in urine. In contrast, the habituationdishabituation task used by Singh et al. (1990) measures the subjects' response to an unconditioned stimulus when its motivational state has not been manipulated.

Our results illustrate the problem associated with using operant conditioning methods to determine a rat's ability to discriminate between odors. The animal's performance may not reflect its behavior in a natural situation. On the other hand, it is also unlikely that preference tests or habituation-dishabituation tasks are truly reflective of an animal's behavior. The use of appropriate experimental techniques, such as comparing performance on subsequent trials when new odors are introduced in an operant task, can differentiate between an animal's natural ability and any artificially induced learning created by the learning paradigm.

The question as to how bacteria contribute to the individual odor of urine remains unanswered. It is possible that metabolic by-products produced by intestinal bacteria interact with a fragment of the MHC glycoprotein in the urine to create a discriminable odor (Singh et al., 1990) or that the immune response genes act indirectly to control the production of a commensal bacterial flora specific to each individual (Howard, 1977). To determine if the MHC plays a direct role in creating a unique odor, the discriminability between urine odors from individuals of 
one strain and urine odors from MHC transgenic individuals of the same strain should be examined.

\section{REFERENCES}

Brown, R. E. (1979). Mammalian social odors: A critical review. Advances in the Study of Behaviour, 10, 103-162.

Brown, R. E., MacDonald, D. W. (Eds.) (1985). Social odours in mammals (Vols. 1-2). Oxford: Clarendon Press.

Brown, R. E., Singh, P. B., \& Roser, B. (1987). The major histocompatibility complex and the chemosensory recognition of individuality in rats. Physiology \& Behavior, 40, 65-73.

Butcher, G. W. HowARD, J. C. (1986). The MHC of the laboratory rat, Rattus norvegicus. In D. M. Weir (Ed.), Handbook of experimental immunology, Vol. 3 (4th ed., pp. 101.1-101.18). Oxford: Blackwell Scientific Publications.

HALPIN, Z. T. (1986). Individual odors among mammals: Origins and functions. Advances in the Study of Behaviour, 16, 39-70.

How ARD, J. C. (1977). H-2 and mating preferences. Nature, 266, $406-408$.

KLEIN, J. (1986). The natural history of the major histocompatibility complex. New York: Wiley.

PENDRY, A. (1984). The use of flexible film isolators for housing experimental animals. Laboratory Animals, 18, 131-134

Pfaff, D., \& PFAFman, C. (1969). Behavioral and electrophysiological responses of male rats to female urine odors. In C. Pfaffman (Ed.), Olfaction and taste (pp. 258-267). New York: Rockefeller University Press.

SCHELINE, R. (1973). Metabolism of foreign compounds by gastrointestinal microorganisms. Pharmacological Reviews, 25, 451-513.

Singh, P. B., Brown, R. E., \& Roser, B. (1987). MHC antigens in urine as olfactory cues. Nature, 327, $161-164$.

Singh, P. B., Brown, R. E., Roser, B. (1989). Soluble classical class I MHC antigens in solution in the body fluids. In P. Ivanyi (Ed.), $M H C+X$ : Complex formation and antibody induction (pp. 226-240). New York: Springer-Verlag.

Singh, P. B., Herbert, J., Roser, B., Arnott, L., Tucker, D. K., \& Brown, R. E. (1990). Rearing rats in a germfree environment eliminates their odors of individuality. Journal of Chemical Ecology, 16, $1667-1682$

Slotnick, B. M., \& Nigrosh, B. J. (1974). Olfactory stimulus control evaluated in a small animal olfactometer. Perceptual \& Motor Skills, 39, 583-597.

SLotnick, B. M., \& PTAK, J. E. (1977). Olfactory intensity-difference thresholds in rats and humans. Physiology \& Behavior, 19, 795-802.

SLotnick, B. M., SChoonover, F. W. (1984). Olfactory thresholds in normal and unilaterally bulbectomized rats. Chemical Senses, 9. $325-340$.

Sundberg, H., Doving, K., Novikov, S., Ursin, H. (1982). A method for studying responses and habituation to odors in rats. Behavioral \& Neural Biology, 34, 113-119.

Yamaguchi, M., Yamazaki, K., BeauchamP, G. K., BARD, J., ThомAS, L., \& Boyse, E. A. (1981). Distinctive urinary odors governed by the major histocompatibility locus of the mouse. Proceedings of the National Academy of Science, USA, 78, 5817-5820.

YAMAZAKI, K., BeAuChamp, G. K., BARD, J., Thomas, L., Boyse, E. A. (1982). Chemosensory recognition of phenotypes determined by the Tla and $\mathrm{H}-2 \mathrm{~K}$ regions of chromosome 17 of the mouse. Proceedings of the National Academy of Science, USA, 79, 7828-7831.

Yamazaki, K. , Beauchamp, G. K., Matsuzaki, O., Kupniewski, D. Bard, J., Thomas, L., \& Boyse, E. A. (1986). Influence of a genetic difference confined to mutation of $\mathrm{H}-2 \mathrm{~K}$ on the incidence of pregnancy block in mice. Proceedings of the National Academy of Science. USA, 83, 740-741.

YamaZAKI, K., BEAUChamP, G. K., Wrsockı, C. J., BARD, J., Thomas, L., Boyse, E. A. (1983). Recognition of $\mathbf{H}-2$ types in relation to the blocking of pregnancy in mice. Science, 221, 186-188.

Yamazaki, K., Boyse, E. A., Mike, V., Thaler, H. T., Mathieson, B. J., Abbott, J., Boyse, E., Zoyas, Z. A., Thomas, L. (1976). Control of mating preferences in mice by genes in the major histocompatibility complex. Joumal of Experimental Medicine, 144, 1324-1335

Yamazaki, K., Yamaguchi, M., Andrews, P., Peake, B., Boyse, E. (1978). Mating preferences of F2 segregants of crosses between MHC congenic mouse strains. Immunogenetics, 6, 253-259.

(Manuscript received October 3, 1990; revision accepted for publication March 5, 1991.) 